$\mathrm{T}$ The catalog. Love it? Hate it? Depending upon who is speaking, it may be cast as the ultimate portal that enables user access to all local and networked resources, or it may be a tool of Byzantine complexity, comprehensible at best to but a small fraction of librarians able to navigate its bibliographic metadata encoded in an arcane 1960s-era format. It is a rich trove of structured and controlled information assembled over decades by the work of countless dedicated catalogers and others. Or, it is the now-obsolete product of a labor-intensive process of description and subject analysis that has no relevance in a Web-centric world where "everything" is findable via the Google search-box. Its attempt to organize knowledge provides catalogers with a raison d'etre, but sends their colleagues and many users fleeing for simpler and more all-encompassing tools. It is our alpha and omega, our yin and our yang.

Few topics in librarianship-perhaps with the conspicuous exception of that perennial library school favorite, our profession's status as a profession-seem to provoke the range and depth of sentiment engendered by discussions of the place of the catalog. Especially in recent years, criticism of the catalog has grown ever more strident, to the point where it has become commonplace in our profession's literature to say that this most basic of library services "sucks." As a consequence, librarians have increasingly fallen into one of two camps, with those critical of the catalog often simplistically characterized as favoring, and those defending it as opposing "change."

A number of initiatives have emerged in response to this ferment. Some of these have focused on our bibliographic metadata, and particularly on its ability to express the relationships and interconnectedness of the bibliographic universe. As we have traditionally cataloged whatever we had "in-hand," our cataloging codes and encoding standards have done a very good job of managing the description of bibliographic items; what they have not generally expressed well are the relationships among items. FRBR and FRAD—-the Functional Requirements for Bibliographic Records and the Functional Requirements for Authority Data-seem promising beginnings for addressing the relationship issues, although there are as-yet very few practical implementations. Resource Description and Access (RDA), the forthcoming successor to AACR2, is designed around FRBR concepts; it will be interesting to see how this plays out in the "real world;" equally interesting will be to what degree the present (or a modified) MARC21 is able to express RDA's FRBR-based relationship model.

Other approaches have focused on developing systems that are able to exploit our existing investment in bibliographic metadata in new and useful ways. The pioneering and best-known example of this, of course, is the discovery tool developed by a partnership of North Carolina State University Libraries and Endeca, which premiered in early 2006. This initiative included several innovative features not previously found in library catalogs, such as search result relevance ranking and the ability to perform faceted searching against a variety of controlledvocabulary indices (subject/topical, form/genre, date, etc.) NCSU's Endeca discovery tool spawned an entirely new product segment for the catalog: major ILS vendors have scrambled to develop their own next-gen products, combining relevancy and facets with additional functionality such as Web 2.0 social and collaborative tools and enhanced federated searching capabilities. The result of all this activity has been the first cross-platform growth opportunity for ILS vendors since the development of resource-linking tools and the ERM.

We at ITAL have watched these trends with keen interest and have published works describing many of the major developments vis-a-vis the catalog in recent years. Indeed, since late 2004, ITAL has published at least eleven major papers on various topics related to improving the catalog. With our publication of Jennifer Bowen's report on the first phase outcomes of the University of Rochester's eXtensible catalog (XC) project in this issue of ITAL, we continue our commitment to publish important research in this area. The Rochester project is noteworthy, both for its modular and metadata-focused approach and for its high visibility as an open source effort that has received significant support from the Andrew W. Mellon Foundation. I predict that this paper will quickly take its place among the other ground-breaking works on the catalog that ITAL has published, and I'll eagerly be awaiting the next progress report on the $\mathrm{XC}$.

\section{"Must-reads" Dept.}

Okay, so I may not be the first out of the gate with this one, but for those of you who haven't looked at it yet, trust me, you'll want to. Jonathan Zittrain's The Future of the Internet and How to Stop It (Yale University Press, 2008), which divides the Internet into "generative" technologies such as the PC, and proprietary appliances such as the iPhone, may or may not resonate with you, but I think it could well become the next big debate about where the Net is and where it should be going. Grab a copy and read it today.

Marc Truitt (marc.truitt@ualberta.ca) is Associate Director, Bibliographic and Information Technology Services, University of Alberta Libraries, Edmonton, Alberta, Canada, and Editor of ITAL. 